\title{
Anesthetic management of a patient with Huntington's chorea -A case report-
}

\author{
Jong-Man Kang, Jun-Young Chung, Jin Hee Han, Yung-Suk Kim, Bong Jae Lee, and Jae-Woo Yi \\ Department of Anesthesiology and Pain Medicine, Kyung Hee University Hospital at Gangdong, College of Medicine, Kyung Hee \\ University, Seoul, Korea
}

Huntington's chorea is a rare hereditary disorder of the nervous system. It is inherited as an autosomal dominant disorder and is characterized by progressive chorea, dementia and psychiatric disturbances. The best anesthetic technique is yet to be established for these patients with increased risk of aspiration due to involvement of pharyngeal muscles and an exaggerated response to sodium thiopental and succinylcholine. The primary goal in general anesthesia for these patients is to provide airway protection and a rapid and safe recovery. We report the anesthetic management of a 51-year-old patient with Huntington's chorea admitted for an emergency operation. (Korean J Anesthesiol 2013; 64: 262-264)

Key Words: Bronchoscope, Huntington disease, Intubation.

Huntington's chorea is a rare autosomal dominant hereditary disorder of the nervous system with complete penetration. The prevalence of the disease is 4-10 per 100,000 in Europe and the United States with no differences between males and females $[1,2]$. The responsible gene is located in chromosome 4 [2]. Pathologically, patients show destruction of neurons in the basal ganglia with most severe atrophy in the caudate nucleus $[1,3]$. The onset of the disease is usually relatively late, at about the age of 30-45 years, and symptoms that begin before the age of 20 occurs in $10 \%$ of affected persons. [1,3]. Death typically occurs 10-30 years after the onset [3]. The symptoms of Huntington's chorea include personality disorder, choreic movements and a progressive decline in cognitive function
[2]. The most ominous motor symptom is dysphagia with dysfunction of pharyngeal muscles, which increases the risk of aspiration. Death is generally due to aspiration complications [3]. The anesthetic management of a patient with Huntington's chorea provides particular challenges. These patients are at an increased risk of pulmonary aspiration [2]. Moreover prolonged apnea, intense shivering and generalized tonic spasm, altered response to the administration of barbiturates and succinylcholine, and increased sensitivity to midazolam are considered high risk perioperative complications $[1,4]$. However, the anesthetic experience with this type of case has not been previously reported in Korea.

Received: April 3, 2012. Revised: 1st, May 8, 2012; 2nd, June 11, 2012. Accepted: June 12, 2012.

Corresponding author: Jae-Woo Yi, M.D., Ph.D., Department of Anesthesiology and Pain Medicine, Kyung Hee University Hospital at Gangdong, College of Medicine, Kyung Hee University, 149, Sangil-dong, Gangdong-gu, Seoul 134-727, Korea. Tel: 82-2-440-6192, Fax: 82-2440-7808, E-mail: mdyjwchk@khu.ac.kr

(c) This is an open-access article distributed under the terms of the Creative Commons Attribution Non-Commercial License (http:// creativecommons.org/licenses/by-nc/3.0/), which permits unrestricted non-commercial use, distribution, and reproduction in any medium, provided the original work is properly cited. 


\section{Case Report}

A 51-year-old male, $50 \mathrm{~kg}$ in weight who had been diagnosed with Huntington's chorea at the 41 years-of-age was admitted for an emergency laparoscopic hernia repair. On physical examination, the patient displayed choreic movements in all limbs, swallowing dysfunction and excessive oral secretions. Laboratory values were all within normal limits, except for leukocytosis. A chest radiograph did not show any abnormal finding and the electrocardiogram (ECG) revealed left ventricular hypertrophy. The patient was transferred to the operating room without being pre-medicated. Routine monitoring included pulse oximetry, ECG, noninvasive blood pressure and end-tidal carbon dioxide and gas monitoring. To check the depth of anesthesia during the operation, A BIS sensor (BISquatro ${ }^{\mathrm{TM}}$, Aspect ${ }^{\circledR}$ Medical Systems, Inc., USA) was attached and measurements were taken when the signal quality index (SQI) was above 95. Muscle relaxation was monitored using an electrical stimulator (Relaxograph $^{\circledR}$, Datex Relaxograph, Finland) by applying the train of four (TOF) on the wrist's ulnar nerve and measuring the contraction of the adductor pollicis muscle. To avoid possible pulmonary aspiration, awake fiberoptic intubation was planned by an expert. The procedure and need to be intubated while awake was explained to the patient. The base of the tongue and pharyngeal walls were anesthetized with $10 \%$ xylocaine spray and $4 \%$ lidocaine was injected trans-tracheally to anesthetise the trachea. The fiberoptic bronchoscope (LF-TP, Olympus, Japan) was checked and loaded with a cuffed endotracheal tube with an inner diameter (ID) of $7.5 \mathrm{~mm}$. The vocal cords were visualized and the tube was passed over the fiberscope into the trachea without difficulty. After confirmation of tracheal intubation by viewing the tube tip inside the trachea using fiberoptics, tube fogging, the inability of the patient to vocalize and end-tidal carbon dioxide, anesthesia was induced with an intravenous computer-controlled infusion pump (Orchestra ${ }^{\circledR}$ Base Primea, Fresenius Vial, France) of $2 \%$ propofol with a target of $4 \mu \mathrm{g} / \mathrm{ml}$ and remifentanil with a target of $4 \mathrm{ng} / \mathrm{ml}$, and an intravenous bolus of $0.5 \mathrm{mg} / \mathrm{kg}$ rocuronium. The radial artery cannula was inserted for invasive blood pressure monitoring. The TOF ratio was zero after induction.

During the surgery, the concentrations of propofol and remifentanil were regulated according to the BIS and mean arterial pressure. Anesthesia was maintained with a TCI of propofol and adequate concentrations to maintain the BIS between 40 and 60 . The mean arterial pressure and heart rate remained within normal limits during the procedure. Four twitches were detected on TOF at 60 and 90 minutes after induction and rocuronium $5 \mathrm{mg}$ was administered each time. The surgery lasted 105 minutes. Ondansetron $4 \mathrm{mg}$ was administered to reduce postoperative nausea and vomiting.
The infusions of remifentanil and propofol were discontinued at the end of the procedure and a neuromuscular blockade was reversed with pyridostigmine $15 \mathrm{mg}$ and glycopyrrolate $0.4 \mathrm{mg}$. Spontaneous respiration and consciousness returned after 9 minutes, at which time the endotracheal tube was removed. The postoperative course was uneventful and there were no complications such as nausea, vomiting or shivering. The patient was transferred to a general ward and discharged from the hospital after 3 uneventful days.

\section{Discussion}

Huntington's disease is a rare neurodegenerative disorder that prior case reports suggest may have implications for the anesthesiologist. Huntington's chorea is a hereditary disease caused by an expansion of Cytosine-Adenine-Guanine repetition in the Huntington gene (HTT: 1T15 gene), resulting in increased production of a mutant protein, Huntington. This protein initially leads to cell loss and atropy, mainly of GABAergic striatal medium spinal output neurons of the caudate, putamen, and cortex [5].

Psychotropic medications, such as antipsychotics, antidepressants, benzodiazepines, and antiepileptics are frequently used for symptom management of this curable condition [5]. So, the anesthesiologist should be aware of potential interactions of these medications with frequently used anesthetics drugs. Moreover, general anesthesia can exacerbate psychiatric symptoms resulting in postoperative agitation, chorea, and psychosis [6].

Pre-medications with metoclopramide and anticholinergics may exacerbate choreiform movements. If anticholinergics must be given, glycopyrrolate, a quaternary ammonium that cannot cross the blood-brain barrier, is preferred over atropine, a tertiary amine that can cross the blood-brain barrier [3].

There are reports that sodium thiopental can cause prolonged apnea. Davies and Blanloeil reported prolonged apnea for a period of $1 \mathrm{hr}$ following induction with sodium thiopental $[7,8]$. But, these cases used more than $5 \mathrm{mg} / \mathrm{kg}$ of sodium thiopental. These doses were likely excessive for sick and debilitated patients and the prolonged effects of sodium thiopental should not be surprising. Others described normal responses to sodium thiopental, so the induction range of $3-5 \mathrm{mg} / \mathrm{kg}$ can be acceptable for use $[3,6]$.

Gualandi and Bonfandi reported a case of prolonged apnea of $2 \mathrm{hr}$ following the administration of $50 \mathrm{mg}$ succinylcholine [9]. Genetic variation of plasma cholinesterase is a potential cause of prolonged muscle paralysis after receiving succinylcholine [6]. In other studies, succinlycholine did not cause prolonged apnea [3]. Despite this, it is possible that muscle paralysis after succinlycholine may be prolonged in patients with Huntington's 
chorea. Therefore, succinylcholine should be used with caution. Short and intermediate duration agents, such as rocuronium and cisatracurium, also appear acceptable for use [6].

Inhalational drugs such as isoflurane, halothane, and sevoflurane have all been advocated and used without problem, but carry the theoretical risks of generalized muscle spasm, prolonged shivering, and protracted recovery periods in which the already compromised airway is at increased risk [2]. To avoid the risk of inhalation agent, using total intravenous anesthesia (TIVA) seems logical in that it allows precise titration of anesthetic, ease of induction, and a rapid and controlled recovery $[2,4]$.

Another anesthetic consideration in these patients centers around the dysphagia that is very common to this group. Dysphagia is probably the most significant motor symptom because it hinders the nutritional intake of those with Huntington's disease and places them at risk for aspiration [3]. Awake fiberoptic intubation has been described as a suitable technique for patients at a high risk of aspiration such as Huntington's disease and appears in some algorithms designed for use in situations where aspiration is a possibility [10]. And, at the termination of the anesthetic, airway reflexes must be also assured to minimize the risk of postoperative aspiration [3].

Spinal anesthesia in a patient with Huntington's chorea also can be used [11]. In our case, we used general anesthesia because spinal anesthesia was impossible due to continuous choreiform movement. We performed an awake intubation with fiberoptic bronchoscope intubation to reduce the risk of pulmonary aspiration by an expert. We used the TIVA technique to avoid the risks that can come with an inhalation agent. For rapid recovery, propofol and remifentanil were selected as the anesthetic agents. Remifentanil is an opioid that, unlike other members of this class of drugs that are metabolized by the liver, undergoes metabolism by nonspecific blood and tissue esterases. It can be administered for a long time at a high dose without accumulation, allowing for rapid recovery and avoiding the risks of prolonged respiratory impairment [2].
We administered an intermediate duration, non-depolarizing neuromuscular blocking drug, rocuronium, and monitored neuromuscular the block by a TOF watch. Using the above techniques, we could successfully anesthetize a patient with Huntington's chorea without perioperative complications.

In conclusion, awake intubation with a fiberoptic bronchoscope can be effective and safe to avoid pulmonary aspiration in a Huntington's disorder. Low dose rocuronium can be used safely and the use of propofol and remifentanil TIVA seem to be a beneficial technique in patients suffering from Huntington disease.

\section{References}

1. Gilli E, Bartoloni A, Fiocca F, Dall'Antonia F, Carluccio S. Anaesthetic management in a case of Huntington's chorea. Minerva Anestesiol 2006; 72: 757-62.

2. MacPherson P, Harper I, MacDonald I. Propofol and remifentanil total intravenous anesthesia for a patient with Huntington disease. J Clin Anesth 2004; 16: 537-8.

3. Cangemi CF Jr, Miller RJ. Huntington's disease: review and anesthetic case management. Anesth Prog 1998; 45: 150-3.

4. Nagele P, Hammerle AF. Sevoflurane and mivacurium in a patient with Huntington's chorea. Br J Anaesth 2000; 85: 320-1.

5. Walker FO. Huntington's disease. Lancet 2007; 369: 218-28.

6. Kivela JE, Sprung J, Southorn PA, Watson JC, Weingarten TN. Anesthetic management of patients with Huntington disease. Anesth Analg 2010; 110: 515-23.

7. Davies DD. Abnormal reponse to anaesthesia in a case of Huntington's chorea. Br J Anaesth 1966; 38: 490-1.

8. Blanloeil Y, Bigot A, Dixneuf B. Anaesthesia in Huntington's chorea. Anaesthesia 1982; 37: 695-6.

9. Gualandi W, Bonfanti G. A case of prolonged apnea in Huntington's chorea. Acta Anaesthesiol 1968; Suppl 6: 235-8.

10. Ovassapian A, Krejcie TC, Yelich SJ, Dykes MH. Awake fibreoptic intubation in the patient at high risk of aspiration. Br J Anaesth 1989; 62: 13-6.

11. Esen A, Karaaslan P, Can Akgün R, Arslan G. Successful spinal anesthesia in a patient with Huntington's chorea. Anesth Analg 2006; 103: 512-3. 